\title{
Potencial de los anillos de crecimiento de Pilgerodendron uviferum para el estudio histórico de las Iglesias de Chiloé, Patrimonio de la Humanidad
}

\author{
The potential use of Pilgerodendron uviferum \\ tree-ring dating in the historical interpretation of the churches of Chiloé, World Heritage
}

\author{
Paulina Puchi a, Ariel A Muñoz a,h *, Mauro E González b,h, Ana Abarzúa c, Katerine Araya d, \\ Ronald Towner ${ }^{\mathrm{e}}$, Reinhard Fitzek ${ }^{\mathrm{a}}$, Andrés Holz ${ }^{\mathrm{f}}$, Daniel Stahle ${ }^{\mathrm{g}}$

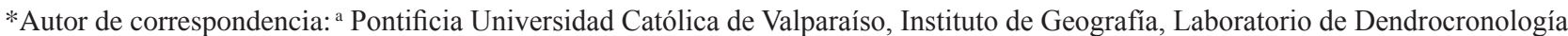 \\ y Estudios Ambientales, Avenida Brasil 2241, Valparaíso, Chile, tel.: 56-32-2274082, arimunoz82@gmail.com \\ ${ }^{\mathrm{b}}$ Universidad Austral de Chile, Instituto de Conservación, Biodiversidad y Territorio, Valdivia, Chile. \\ ${ }^{c}$ Universidad Austral de Chile, Instituto de Ciencias Ambientales y Evolutivas, Valdivia, Chile. \\ ${ }^{\mathrm{d}}$ Fundación Amigos de las Iglesias de Chiloé, Ancud Chile. \\ e University of Arizona, Laboratory of Tree-Ring Research, Arizona, Estados Unidos. \\ ${ }^{\mathrm{f}}$ Portland State University, Department of Geography, Portland, Estados Unidos. \\ g University of Arkansas, Estados Unidos.

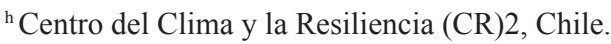

\begin{abstract}
SUMMARY
The churches on Chiloé Island are the oldest wood structures in Chile. Chiloé islanders adapted European colonial techniques and developed unique regional construction styles when building these UNESCO-recognized community and religious centers. Although these historical treasures are preserved, much of the construction history of these churches remains unknown. Tree-ring dating is a proven archeological dating method used to identify the logging dates of wood used for constructiong historical buildings. The majority of churches on Chiloé were constructed using Pilgerodendron uviferum ("ciprés de las Guaitecas") wood. Therefore, the objective of this study was to evaluate the potential use of this species to date timber found at churches in Vilupulli and Ichuac. Timber sections from both structures were successfully cross-dated using three regional multi-century long P. uviferum tree-ring site chronologies located in the Chiloé region (two from the island and one from the nearby Andes region). Samples from pillars located in the tower of the Vilupulli church contained 311 and 181 tree-rings, respectively, while four samples from floor beams in the Ichuac church contained 79, 89, 97 and 135 annual growth. Timber used to build the Vilupulli church tower cross-dates to 1918 and corroborates colloquial knowledge that the structure was built in the early 20th century. Dates obtained from the floor beams in theIchuac church range from 1920 to1929, and contradict the colloquial thinking that the structure was built at the end of the 19th century, though these could represent material used in a later restoration not previously recorded in Ichuac's local history. These findings confirm that $P$. uviferum presents strong capabilities for further use in tree ring dating of important historical structures located in the temperate region of Southern Chile.
\end{abstract}

Key words: dendroarchaeology, historical structures, Pilgerodendron uviferum.

\section{RESUMEN}

Las iglesias de Chiloé son antiguas estructuras de madera reconocidas patrimonio de la humanidad por la UNESCO. Gran parte de su historia de construcción y reparaciones aún se desconoce. Considerando que muchas de las iglesias de Chiloé fueron construidas utilizando madera de Pilgerodendron uviferum, el objetivo de este trabajo fue evaluar el potencial de esta especie para datar piezas de madera de dos de estas históricas construcciones: las iglesias de Vilupulli e Ichuac. En Vilupulli se dataron piezas de 311 y 181 años provenientes de los pilares de la torre. Estas piezas fueron fechadas con cronologías de ancho de anillos de $P$. uviferum cercanas a las dos iglesias. También utilizando estas cronologías se dataron piezas de 79, 89, 97 y 135 años obtenidas a partir de los pilotes que sostienen el piso de la iglesia de Ichuac. Considerando que Vilupulli fue construida a principios del siglo XX, es posible que las muestras de la torre que presentaron fechas cercanas a 1918, sean parte del proceso tardío de construcción de la iglesia o de una restauración posterior. Por su parte, Ichuac fue construida a finales del siglo XIX, por lo que las piezas del piso que dataron entre 19201929 , formarían parte de una posible restauración no descrita previamente en archivos históricos, la cual pudo ocurrir incluso varios años posterior a la fecha del anillo más reciente encontrado en las piezas estudiadas. Se concluye que $P$. uviferum tiene alto potencial para estudios históricos en estructuras patrimoniales en el sur de Chile.

Palabras clave: dendroarqueología, estructuras patrimoniales, Pilgerodendron uviferum. 


\section{INTRODUCCIÓN}

Una de las aplicaciones más notables del estudio de los anillos de crecimiento de los árboles, conocido como dendrocronología, es su uso para la datación de asentamientos humanos, obras de arte, instrumentos musicales y construcciones históricas, rama que se ha denominado dendroarqueología (Baillie 1995, Kuniholm et al. 2001).

Dataciones dendroarqueológicas de construcciones históricas tales como iglesias, casas, barcos y asentamientos humanos han sido desarrollados principalmente en Norteamérica y Europa (Kuniholm et al. 2001, Slayton et al. 2009, Barclay y Rayburn 2014). En el caso de Sudamérica, solo dos estudios dendroarqueológicos han sido publicados, uno en el altiplano boliviano de los Andes Centrales y el otro en el desierto de Atacama (Rivera et al. 2010, Morales et al. 2013). En estos lugares, el frío y la aridez permiten la preservación de madera sub-fósil durante varios siglos. Por el contrario, en climas templados lluviosos, las condiciones adversas del ambiente impiden que las construcciones de madera permanezcan por mucho tiempo, ya que la mayoría de la madera expuesta a ambientes húmedos tiende a descomponerse rápidamente (Morales et al. 2013).

En el sur de Chile existen dos especies de coníferas endémicas que han sido utilizadas históricamente para la construcción de casas, barcos e iglesias dada la alta durabilidad de sus maderas. Una de ellas es Pilgerodendron uviferum (D. Don) Florin (ciprés de las Guaitecas), considerada la conífera de distribución más austral del mundo $\left(39^{\circ} 35^{\prime} \mathrm{S}-55^{\circ} 30^{\prime} \mathrm{S}\right)$ y que puede llegar a vivir hasta 880 años (Bannister et al. 2012). La otra especie es Fitzroya cupressoides (Molina) I.M. Johnst. (alerce) (39 $50^{\circ}$ - $43^{\circ}$ $\mathrm{S})$, la cual es la segunda especie más longeva del mundo y la primera en Sudamérica (Veblen et al. 2005, Lara et al. 2013). Ambas especies habitan lugares donde la precipitación supera $2000 \mathrm{~mm}$ al año, pudiéndose encontrar bosques de estas especies en zonas en donde la precipitación anual muestra un rango de 5.000 a $8.000 \mathrm{~mm}$ en algunos casos (Donoso et al. 2013, Lara et al. 2013). A pesar de la alta precipitación, el crecimiento de estas especies ha demostrado tener un ritmo anual sensible a la estacionalidad de la precipitación y la temperatura, siendo útil para reconstrucciones climáticas (Lara et al. 2008) y también para el estudio de la dinámica de establecimiento y el régimen de disturbios en los bosques que habitan (Bannister et al. 2008, Donoso et al. 2013). No obstante, hasta la fecha ninguna de estas especies ha sido utilizada para la datación de estructuras patrimoniales, ni de asentamientos humanos históricos o pre-históricos en Sudamérica.

En el sur de Chile, la madera de P. uviferum fue empleada en el pasado para la manufactura de muelles, construcciones navales, postes, tejuelas y muebles (Lara et al. 2013). Es importante destacar la notable resistencia a la descomposición de P. uviferum (Solis et al. 2004), considerando la pluviosidad de la región donde crece y la hu- medad de los sitios donde habita, en su mayoría turberas de Sphagnum sp. en la isla de Chiloé (Lara et al. 2013).

Las estructuras internas de muchas de las iglesias de Chiloé, tales como las vigas, pilares y pilotes fueron construidas a través de la historia principalmente con madera de P. uviferum, mientras que en la mayoría de los casos para el revestimiento de las iglesias se utilizaron tejuelas de F. cupressoides (Montecinos et al. 1995). Las iglesias de Chiloé son uno de los principales patrimonios arquitectónicos de Chile, debido a que el diseño de estas construcciones combinan las habilidades de los carpinteros locales, colonos y religiosos europeos quienes habitaron la Isla de Chiloé desde el siglo XVI. Los religiosos proporcionaron las técnicas de construcción y el diseño inspirado en las iglesias de sus países, mientras que los carpinteros chilotes aportaron la mano de obra, materiales (madera) y sus propias técnicas, desarrolladas a partir de la construcción de sus botes (Guarda 1984, Montecinos et al. 1995).

Las iglesias chilotas fueron fundamentales en la formación de las ciudades y cultura de los habitantes de Chiloé, por consiguiente, actualmente se pueden encontrar decenas de iglesias de madera distribuidas en la isla y sus archipiélagos (Montecinos et al. 1995, Berg 2007, Sahady et al. 2007). El incalculable valor cultural de las iglesias de Chiloé se debe a su relación con la cultura local y al sistema único de construcción basado en ensambles de madera nativa, los cuales son reforzados con tarugos y escaso uso de clavos. Por todo lo anterior, en el año 2003 la UNESCO declaró Patrimonio de la Humanidad a 16 iglesias de Chiloé, promoviendo su restauración y conservación.

Hasta la fecha, la mayoría de las iglesias de Chiloé han sido datadas a través de registros históricos, principalmente basados en las cartas que extendían los sacerdotes a la Iglesia en Europa, informándoles acerca de las necesidades de reparación o instalación de una estructura en particular (Guarda 1984, Montecinos et al. 1995). Archivos de la iglesia y archivos locales, como diarios o fotografías, se volvieron más frecuentes y exactos en la segunda mitad del siglo XX, favoreciendo un más detallado registro de la historia. Previo a esto, los documentos disponibles en muchos casos no dejan claro el año exacto de construcción o de las restauraciones posteriores de las iglesias, tampoco el tiempo que duraron estos procesos. Por esta razón, el uso de otras fuentes de información, como las provistas por la datación dendrocronológica, aplicada a una gran cantidad de piezas de madera de las estructuras, podría proveer de información valiosa para la historia de la fundación y restauración de las iglesias de Chiloé.

En la actualidad, hay valiosas contribuciones de los habitantes por mantener vigente sus tradiciones religiosas y de construcción, lo cual se suma al esfuerzo de diferentes grupos de investigadores que están trabajando en la reconstrucción de la historia de las iglesias de Chiloé. Sin embargo, la información recolectada acerca de las fechas de construcción, restauración y remodelaciones a lo largo de la vida de las estructuras aun es limitada. Hasta la fecha, 
los métodos para datar estas estructuras se fundamentan en documentación histórica, a pesar de que las iglesias de Chiloé hayan sido construidas casi en su totalidad con piezas de maderas de especies nativas que han mostrado potencial dendrocronológico. Por lo anterior, la datación de piezas de madera de las iglesias a través del estudio de los anillos de crecimiento de los árboles utilizados en su construcción, podría generar información cuantitativa sobre las fechas de intervención de estas construcciones, en las cuales, la evidencia histórica es escasa y las fechas asignadas pueden ser en muchos casos especulativas (Guarda 1984).

Considerando que $P$. uviferum ha sido la especie mayormente utilizada en las iglesias de Chiloé y en otras obras patrimoniales en la isla y sus alrededores (Guarda 1984, Montecinos 1995), junto a la aptitud dendrocronológica que ha mostrado en estudios ecológicos y climáticos (Lara et al. 2008, Banister et al. 2012), la hipótesis de este estudio es que las estructuras de madera de esta especie tienen alto potencial dendroarqueológico. A partir de lo anterior, el objetivo de este estudio es evaluar en forma exploratoria el potencial de $P$. uviferum para la datación de piezas de ma- dera en las iglesias de Ichuac y Vilupulli, ambas ubicadas en la Isla de Chiloé. El uso de dataciones dendroarqueológicas permitiría contar con un nuevo registro histórico que abriría una nueva oportunidad para precisar la historia y resaltar la importancia de estas estructuras de madera, que se cree son de las más antiguas del hemisferio sur.

\section{MÉTODOS}

Área de estudio. Las iglesias de Vilupulli (VLP) e Ichuac (ICH) se encuentran ubicadas en el Isla de Chiloé en las comunas de Chonchi y Puqueldón, entre los $42^{\circ} 6^{\prime} \mathrm{S} 73^{\circ} 8^{\prime} \mathrm{O}$ y $42^{\circ} 61^{\prime} \mathrm{S} 73^{\circ} 73^{\prime} \mathrm{O}$, respectivamente (figura 1). Considerando un rango latitudinal más extenso, $40^{\circ} 08^{\prime} \mathrm{S}$ y $43^{\circ} 59^{\prime} \mathrm{S}$, se ubicó el conjunto completo de cronologías de ancho de anillo de P. uviferum analizadas como series de referencia en este estudio, incluyendo aquellas que fueron evaluadas y no involucradas en los análisis (cuadro 1). Los sitios Piuché (ETP), Chaiguata (CHA) y Tepuhueico (TEP) se localizaron en la Isla de Chiloé, mientras que las cronologías Santa Lucía (SLN) y Puerto Raúl Marín Balmaceda

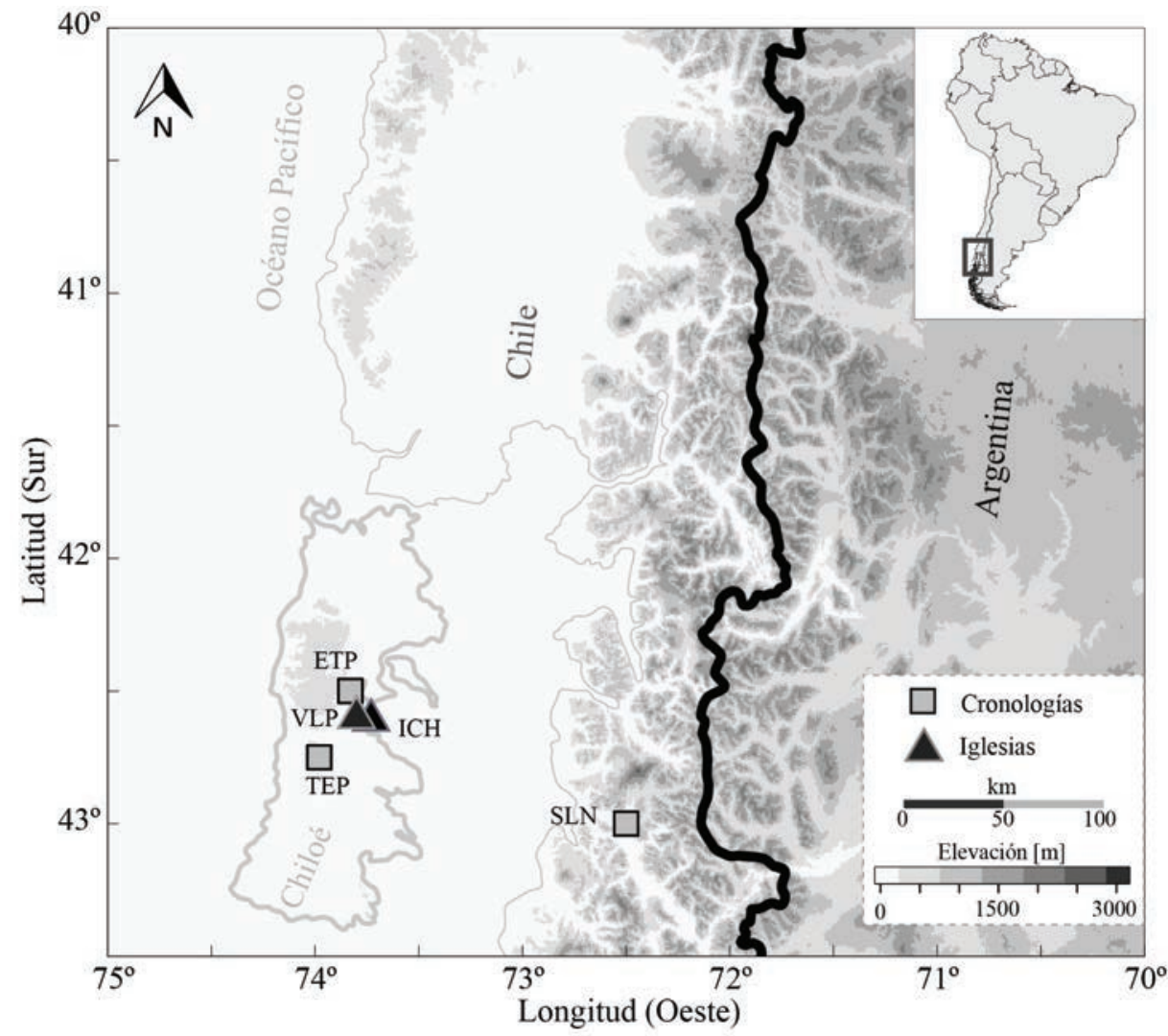

Figura 1. Ubicación geográfica de la Isla de Chiloé y de las iglesias históricas de Vilupulli (VLP) e Ichuac (ICH). Se incluye la ubicación de las cronologías de ancho de anillo de P. uviferum de los sitios Piuche (ETP), Tepuhueico (TEP) y Santa Lucía (SLN). El modelo de elevación digital resalta el gradiente de altura en un rango de 16 m s.n.m (color blanco) hasta los 3.289 m s.n.m (color negro).

Location of Chiloé Island, historic churches of Vilupulli (VLP) and Ichuac (ICH), and Pilgerodendron uviferum tree-ring site collections at Piuché (ETP), Tepuhueico (TEP) and Santa Lucía (SLN). Elevation ranges from $16 \mathrm{~m}$ a.s.l. (white) to 3,289 m a.s.l. (black), as depicted in a digital elevation model (DEM). 
(PMR) provienen de sitios ubicados en Chile continental, cerca de la Isla de Chiloé, a excepción de Hueicolla (HUE) ubicada dos grados de latitud al norte de la isla (cuadro 1).

La isla de Chiloé se caracteriza por presentar un clima templado marítimo lluvioso, con temperaturas medias anuales que fluctúan entre $10,2{ }^{\circ} \mathrm{C}$ y $10,6^{\circ} \mathrm{C}$, mientras que la máxima promedio del mes más cálido (febrero) varía entre $17,8^{\circ} \mathrm{C}$ y $19,4{ }^{\circ} \mathrm{C}$, y la mínima promedio entre 3,2 ${ }^{\circ} \mathrm{C}$ y $4,7{ }^{\circ} \mathrm{C}$ en los meses más fríos del invierno, julio y agosto. Las precipitaciones están presentes durante todo el año, las cuales varían en un rango de 2.000 a $3.000 \mathrm{~mm}$ anuales en la costa occidental de la Isla Grande de Chiloé (Di Castri y Hajek 1976) y pueden alcanzar más de 4.000 mm anuales en sectores altos de la Cordillera de la Costa dentro de la isla.

Series de referencia. Para este estudio se evaluaron seis cronologías de ancho de anillos de P. uviferum como series de referencia para datar las muestras provenientes de las dos iglesias (cuadro 1). Tres de las cronologías se extrajeron del International Tree-Ring Data Bank (ITRDB 2014) y las tres restantes fueron desarrolladas por Holz y Veblen (2011). Los sitios se ubicaron a elevaciones entre 5 y 800 m s.n.m. (cuadro 1). Para evaluar las relaciones de las cronologías seleccionadas con la variabilidad climática, se utilizaron datos climáticos grillados (CRUTS3.23) desde el servidor KNMI administrado por el The Royal Netherlands Meteorological Institute (KNMI 2015). Este análisis se utilizó para evaluar el grado de similitud entre cronologías y sus señales climáticas.

De las seis cronologías de ancho de anillo de P. uviferum utilizadas para este estudio, solo tres presentaron buenos estadísticos de datación cruzada con las muestras de las iglesias de Vilupulli e Ichuac (cuadro 2). Utilizando series de crecimiento de estas tres cronologías individuales, se desarrolló una cronología compuesta (COM) con el objetivo de representar una señal regional de los tres sitios (cuadro 2). Las cronologías Piuché (ETP) y Santa Lucía (SLN) tuvieron longitudes de 433 y 497 años, respectivamente, y alcanzaron valores de EPS $>0,85$ por más 300 años en ambos casos. Por su parte, la cronología Tepuhueico (TEP) alcanzó una extensión temporal de 487 años, sin embargo alcanzó valores de EPS $>0,85$ en un periodo de 160 años. El estadístico EPS es utilizado para discriminar en la robustez de la señal común (promedio temporal) de una cronología en cuanto a varianza y número de árboles, al ser comparada con una cronología teórica perfectamente replicada (Briffa 1995). También fue la cronología Tepu-

Cuadro 1. Ubicación geográfica de las cronologías de ancho de anillos de Pilgerodendron uviferum utilizadas en este estudio. Characteristics of the Pilgerodendron uviferum tree-ring collection site.

\begin{tabular}{lcccccc}
\hline Sitios & Código & Periodo & Latitud (S) & $\begin{array}{c}\text { Longitud } \\
(\mathrm{O})\end{array}$ & $\begin{array}{c}\text { Elevación } \\
\text { (m s.n.m) }\end{array}$ & Fuente $^{\mathrm{C}}$ \\
\hline Hueicolla & HUB & $1868-1975$ & $40^{\circ} 08^{\prime}$ & $73^{\circ} 31^{\prime}$ & 800 & ITRDB CHIL014 \\
Piuché & ETP & $1554-1987$ & $42^{\circ} 30^{\prime}$ & $73^{\circ} 50^{\prime}$ & 750 & ITRDB CHIL018 \\
Santa Lucía & SLN & $1489-1986$ & $43^{\circ} 00^{\prime}$ & $72^{\circ} 30^{\prime}$ & 540 & ITRDB CHIL017 \\
Pto. Raúl Marín & PRM & $1741-2005$ & $43^{\circ} 59^{\prime}$ & $72^{\circ} 42^{\prime}$ & 5 & Holz y Veblen 2011 \\
Chaiguata & CHA & $1770-2005$ & $43^{\circ} 03^{\prime}$ & $73^{\circ} 54^{\prime}$ & 191 & Holz y Veblen 2011 \\
Tepuhueico & TEP & $1518-2005$ & $42^{\circ} 45^{\prime}$ & $73^{\circ} 59^{\prime}$ & 185 & Holz y Veblen 2011 \\
\hline
\end{tabular}

${ }^{\mathrm{C}}$ ITRDB (2014).

Cuadro 2. Características de las cronologías de Pilgerodendrum uviferum utilizadas para la datación de las piezas de madera de las Iglesias de Ichuac y Vilupulli.

Statistical characteristics of the Pilgerodendron uviferum chronologies used in this study to cross-date timber sections from the churches of Ichuac and Vilupulli.

\begin{tabular}{cccccc}
\hline Código & Periodo & $\mathrm{N}^{\circ}$ de muestras & Sensibilidad media & Intercorrelación entre series & EPS $(>0,85)$ \\
\hline ETP & $1554-1987$ & 27 & 0,216 & 0,595 & 1610 \\
SLN & $1489-1986$ & 60 & 0,249 & 0,574 & 1670 \\
TEP & $1518-2005$ & 17 & 0,199 & 0,515 & 1845 \\
COM & $1553-2005$ & 100 & 0,234 & 0,490 & 1735 \\
\hline
\end{tabular}

*COM: cronología compuesta de ETP, SLN y TEP. 
hueico (TEP) la que presentó la menor sensibilidad media de las tres analizadas (cuadro 2). Este estadístico es utilizado para evaluar la variación interanual en una cronología e indica su capacidad potencial para mostrar variaciones en el crecimiento de un año con respecto a otro (Cook y Kairiukstis 1990, Briffa 1995). En las tres cronologías individuales (ETP, SLN, TEP) y en la cronología compuesta por cien series de los tres sitios (COM1), la intercorrelación entre las series de crecimiento de $P$. uviferum fue $\mathrm{r}>$ 0,49 (cuadro 2). Este estadístico es utilizado para describir el grado de similitud entre las muestras de una cronología (Holmes 1983, Cook y Kairiukstis 1990).

Piezas de madera de la Iglesia de Vilupulli. La iglesia de Vilupulli fue restaurada entre agosto 2004 y octubre 2005. Las restauraciones de las iglesias Patrimonio de la Humanidad de Chiloé se llevan a cabo identificando cada pie- za según especie y ubicación en la estructura, las cuales son evaluadas según su estado de conservación. Esto se realiza al evaluar el nivel de pudrición y las capacidades físico-mecánicas de las piezas de madera. Las piezas que no cumplen con las características deseadas son reemplazadas por nuevas piezas de la misma especie. En muchos casos, las piezas reemplazadas son donadas a la comunidad aledaña y en otros casos son almacenadas en bodegas o en centros de difusión, como el de la Fundación Amigos de las Iglesias de Chiloé (2015), institución encargada del desarrollo de estas restauraciones. En el caso de la Iglesia de Vilupulli, las piezas que fueron reemplazadas en la restauración de la torre y de otros elementos arquitectónicos de la estructura, fueron almacenadas en un galpón en frente de la iglesia. En este lugar, se hallaron dos pilares de la torre (figura 2A y 2B), de los cuales se colectaron secciones transversales o rodelas (figura 2C), que fue-

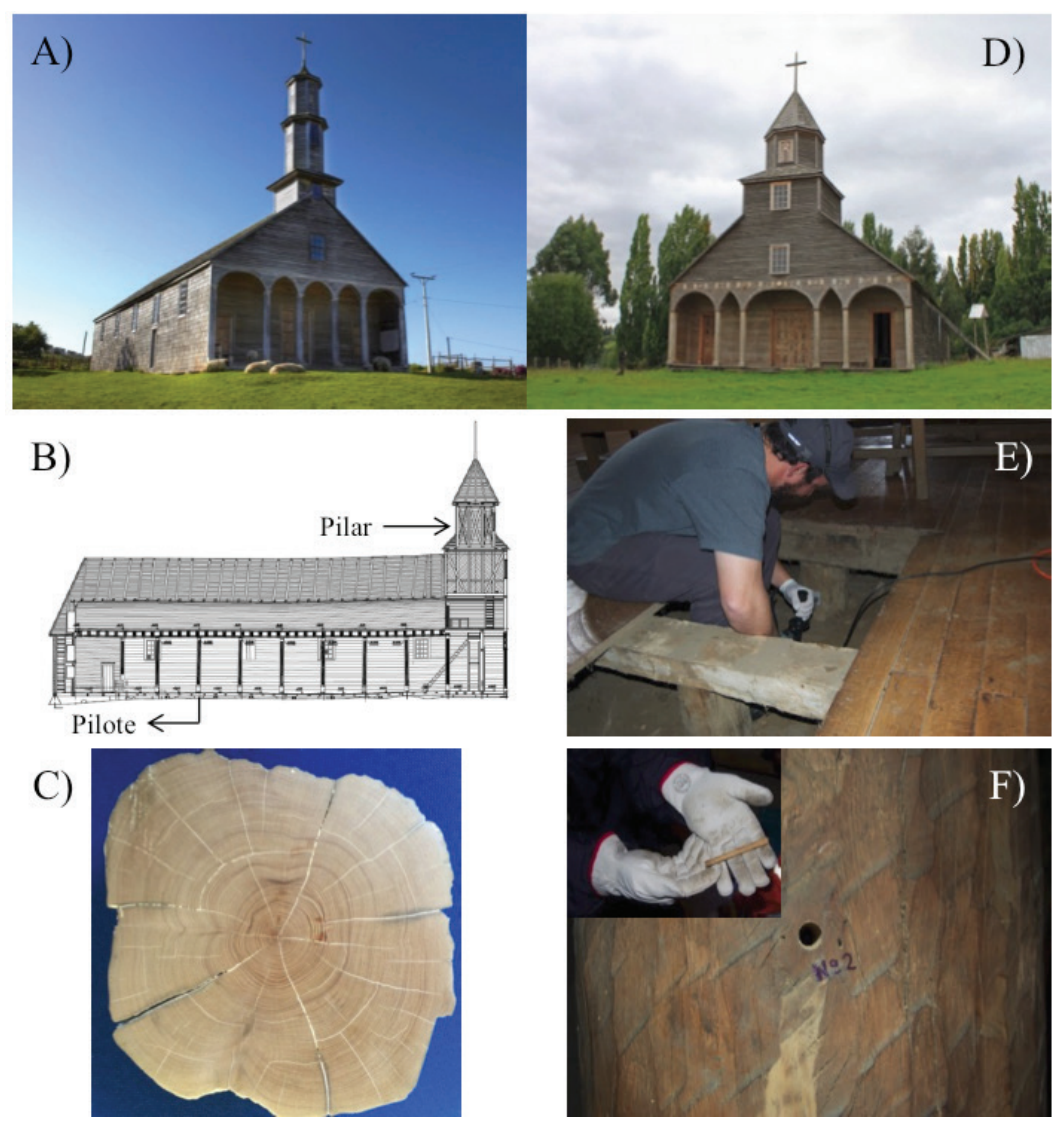

Figura 2. A) Iglesia de Vilupulli. B) Plano tradicional del corte longitudinal de las Iglesias de Chiloé, indicando la ubicación del muestreo del pilar de la iglesia de Vilupulli y del pilote del piso de la iglesia de Ichuac. C) Corte transversal de pieza de madera de $P$. uviferum obtenida del pilar de la torre principal de la iglesia de Vilupulli durante el periodo de restauración. D) Iglesia de Ichuac. E) Toma de muestras de los pilotes del piso de la iglesia de Ichuac. F) Tarugo de madera de P. uviferum obtenido de los pilotes del piso de la iglesia de Ichuac.

A) Church of Vilupulli. B) Schematic of the general architectural design of churches in Chiloé indicating the location of pillar sampling of Vilupulli Church and pile floor Ichuac Church. C) Cross-sectional view of a P. uviferum timber sample obtained from a pillar salvaged from the tower of the church in Vilupulli during restoration. D) Church of Ichuac. E) Coring a floor beam at the Ichuac church. F) Tree-ring core sample obtained from a floor beam from the Ichuac church. 
ron procesadas en el Laboratorio de Dendrocronología y Cambio Global de la Universidad Austral de Chile, y en el Laboratorio de Dendrocronología y Estudios Ambientales del Instituto de Geografía de la Pontificia Universidad Católica de Valparaíso.

Piezas de madera de la Iglesia de Ichuac. Utilizando un taladro eléctrico con una broca arqueológica, se extrajeron muestras de cuatro pilotes que sostenían el piso de la Iglesia de Ichuac (figura 2D, 2E y 2F). Las muestras extraídas se rotularon y posteriormente fueron procesadas en los mismos laboratorios de Valdivia y Valparaíso.

Datación de piezas de madera de las iglesias. Las muestras (rodelas) de la iglesia de Vilupulli fueron lijadas progresivamente con distintas granulometrías (secuencia de granos grueso a fino), hasta que los anillos de crecimiento de $P$. uviferum fueron claramente distinguibles. Las muestras (tarugos de incremento) de la iglesia de Ichuac fueron montadas en molduras de madera y lijadas de la misma forma que las rodelas de la iglesia de Vilupulli. Posteriormente las muestras fueron medidas en laboratorio utilizado el sistema VELMEX diseñado para el estudio de anillos de árboles, el cual permite obtener mediciones de los crecimientos anuales con resolución de micrones $(0,001 \mathrm{~mm})$. La datación de las muestras (asignación de un año calendario a cada anillo) fue guiada por el programa COFECHA (Holmes 1983). Este software permite asignar años probables a las muestras en las que se desconoce la datación. Esto se lleva a cabo a través de la comparación del patrón de crecimiento de la muestra no-datada, con los patrones de crecimiento de series de referencia correctamente datadas en el año calendario. Estas series de referencia corresponden a cronologías de ancho de anillos de sitios individuales o pueden ser construidas con series de crecimiento de varios sitios. Las cronologías de ancho de anillos utilizadas como series de referencia para datar piezas de madera, representan el crecimiento promedio de un grupo de árboles de la misma especie, idealmente cercanos al sitio de estudio. Por esto, uno de los supuestos de este tipo de estudios es que la madera que se busca datar proviene de un lugar similar en condiciones ambientales, aproximadamente cercano o en algunos casos incluso del mismo lugar de los árboles que componen la cronología o serie de referencia.

Una evaluación gráfica de las correlaciones indicadas por el software COFECHA se llevó a cabo estandarizando las series de crecimiento de las piezas de madera de las iglesias y de las cronologías, utilizando una función de línea recta o una curva exponencial negativa. Este procedimiento de estandarización fue utilizado para remover el efecto de la edad en las series de crecimiento. Lo anterior se llevó a cabo en el programa ARSTAN, el cual permite remover las tendencias de las series de crecimiento inducidas por la edad del árbol y por las influencias no-climáticas, propias de la dinámica del rodal (Cook y Kairiukstis 1990). Las cronologías y las series de crecimiento de las piezas de las iglesias fueron posteriormente expresadas como índice de ancho de anillos, el cual es una representación adimensional del crecimiento que favorece la comparación entre cronologías.

Establecimiento de fecha de corte. Para la determinación del año de corte de las muestras dendroarqueológicas de las iglesias de Chiloé, se utilizó la metodología propuesta por Nash (1999), el cual definió la fecha de corte del árbol en relación a la presencia o ausencia de la corteza o albura, y a los atributos de calidad del anillo más externo junto a la corteza. Los aspectos considerados fueron:

a) Fecha precisa al año de corte. Muestra con corteza presente y presencia del último anillo (año) de crecimiento, correspondiente a la fecha de muerte del árbol.

b) Fecha de corte. El anillo exterior, que corresponde al anillo más reciente al momento de la muerte del árbol, se encuentra intacto y la madera externa presenta una curvatura suave sugiriendo que sobre ella se encontraba la corteza.

c) Fecha cercana de corte. Calidad de anillos exteriores y presencia de xilema activo (albura), sugiere que la fecha se encuentra a unos pocos años de cuando el árbol murió. d) Fecha de corte indeterminada. No se puede identificar cuan lejos está del último anillo o anillo más externo en el tronco.

Adicionalmente, para estimar un posible número de anillos faltantes en las muestras de $P$. uviferum que presentaban albura, se analizaron series de crecimientos provenientes de tres cronologías desarrolladas por los autores en zonas cercanas a las iglesias de Ichuac y Villopulli. De las tres cronologías, solamente una de ellas presentó series de crecimiento con presencia clara de albura. Utilizando estas muestras se estimó el número de anillos potencialmente presentes en la albura.

\section{RESULTADOS}

Datación de piezas de madera de la iglesia de Vilupulli. La muestra del pilar de la torre codificada como VLP-05, que presentó 311 años, fue datada para el periodo 1608-1918 con las cronologías Piuché (ETP), Tepuhueico (TEP) y Santa Lucía (SLN) con correlaciones de $r=0,44(P<0,05)$, $\mathrm{r}=0,40(P<0,05)$ y $\mathrm{r}=0,30(P<0,05)$, respectivamente. La muestra VLP-05 presentó correlaciones similares con la cronología compuesta (COM1) (figura 3, cuadro 3). La comparación gráfica de las tres cronologías de ancho de anillos con los crecimientos medidos en VLP-05 proveniente de los pilares de la iglesia de Vilupulli, evidenció la similitud de los crecimientos medidos en la muestra y las series de referencia (figura 3), corroborando lo sugerido por las correlaciones indicadas por el programa COFECHA. La ausencia de corteza en esta muestra no permitió asignar un año calendario exacto a la fecha de corte de los árboles. Sin embargo, la presencia de albura permitió estimar que la fecha de corte podría estar cercana al año 1918, 
fecha del último anillo presente en la muestra (cuadro 3). El número de anillos promedio en muestras cofechadas provenientes de árboles vivos con albura fue de 20 a 50 años, siendo 30 años el promedio, por lo cual una fecha cercana a 1918 a partir de la muestra con albura de Vilupulli debe ser interpretada con precausión. Por su parte, al último anillo encontrado en la muestra VLP-01 se le asignó el año 1866 de acuerdo a las correlaciones con las series de referencia. La ausencia de corteza y albura visible, no permitió asignar una fecha probable de corte a los árboles que dieron origen a esta pieza de madera (cuadro 3).

La otra muestra del pilar de la torre de la iglesia de Vilupulli VLP-01, que alcanzó una extensión temporal de 181 años, presentó los mejores estadísticos de datación cruzada con la cronología Tepuhueico (TEP) logrando una correlación de $\mathrm{r}=0,34(P<0,05)$ (cuadro 3, figura 4). Vi-

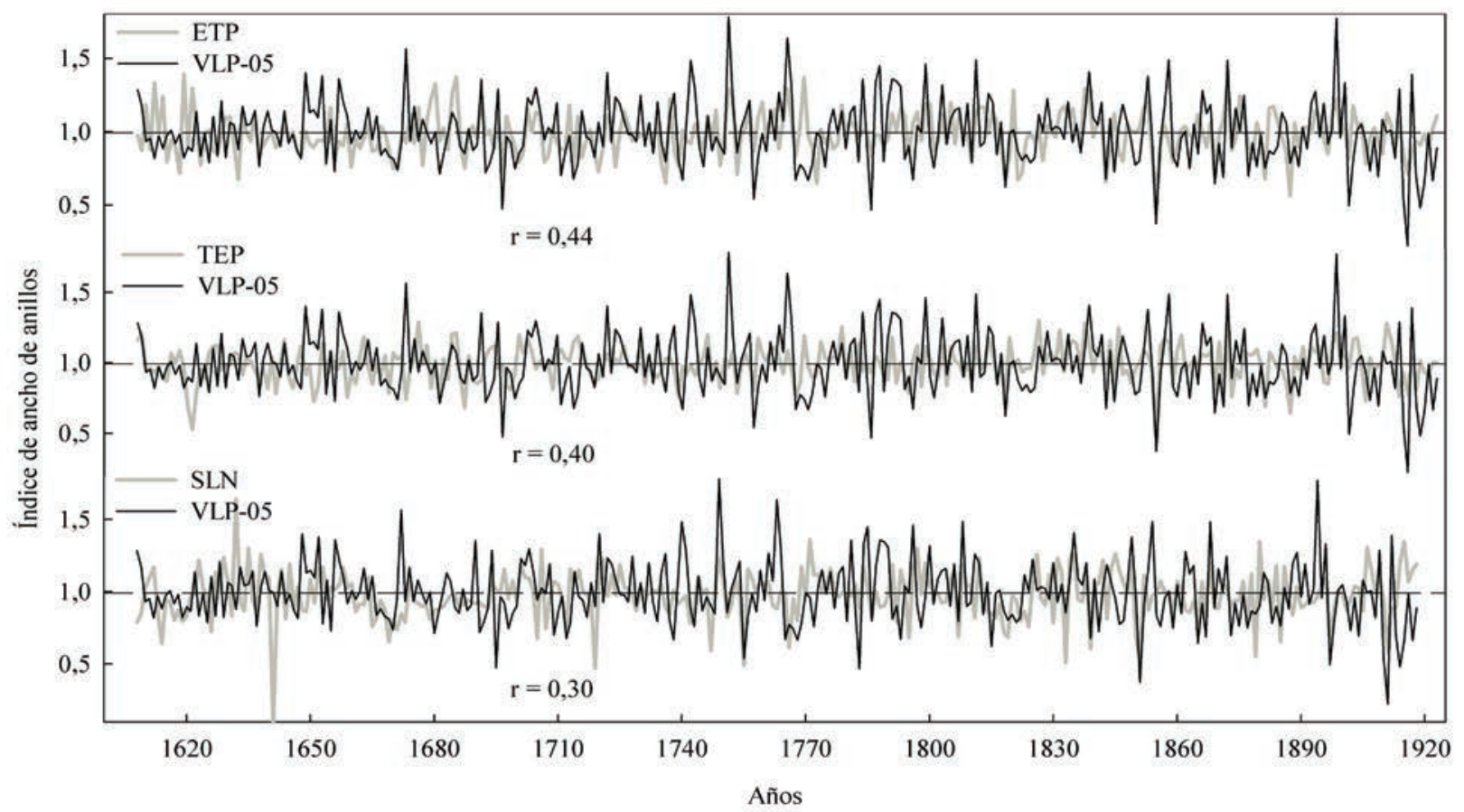

Figura 3. Series de tiempo de trescientos años de comparación entre el pilar de la torre de la Iglesia de Vilupulli VLP-05 (línea negra) con tres cronologías estándares de P. uviferum Piuche, Tepuhueico y Santa Lucia (ETP, TEP, SLN; línea gris) para el periodo de 16081918. Nótese la correspondencia temporal de la variabilidad interanual entre el pilar VLP-05 y las cronologías de ancho de anillos ETP, SNL y TEP. Se incluyen los coeficientes de correlación de Spearman.

Time-series comparison (and Spearman correlation coefficients) between Vilupulli's church tower pillar sample VLP-05 (plotted in black) and three different P. uviferum standard chronologies; Piuché (ETP), Tepuhueico (TEP) and Santa Lucía (SLN) (plotted in gray), over the $1608-1918$ period. Note strong visual agreement in high-frequency inter-annual variability between the VLP-05 pillar ring-widths and the regional tree-ring chronologies at ETP, SNL and TEP.

Cuadro 3. Características de las dataciones de los pilares de la iglesia de Vilupulli.

Tree-ring dating and statistical characteristics of the pillar timber section from the Vilupulli tower.

\begin{tabular}{ccccccc}
\hline Código & N $^{\circ}$ de años & Año de inicio & Año de término & Anillo externo* & Cronología & $\mathrm{r}$ \\
\hline VLP-05 & 311 & 1608 & 1918 & C & ETP & 0,44 \\
VLP-05 & 311 & 1608 & 1918 & C & TEP & 0,40 \\
VLP-05 & 311 & 1608 & 1918 & C & SLN & 0,30 \\
VLP-05 & 311 & 1608 & 1918 & C & COM & 0,36 \\
VLP-01 & 181 & 1685 & 1866 & D & TEP & 0,34 \\
VLP-01 & 181 & 1685 & 1866 & D & COM & 0,23 \\
\hline
\end{tabular}

*Establecimiento de fecha de corte (Nash 1999). 
sualmente presentó algunas diferencias respecto del patrón observado en la cronología TEP, presentando una mayor amplitud al comienzo de la serie de crecimiento con respecto a lo observado en la cronología (figura 4). La misma fecha se obtuvo al comprar esta serie de crecimiento con la cronología compuesta (COM), aunque con menores valores en los estadísticos de datación (cuadro 3 ).

Datación de piezas de madera de la iglesia de Ichuac. Las series de crecimiento de los cuatro pilotes del piso de la Iglesia de Ichuac presentaron aceptables estadísticos de datación cruzada con las tres cronologías de ancho de anillos de P. uviferum (cuadro 4). Menores valores de correlación se presentaron entre los pilotes y la cronología compuesta (COM) por árboles de las tres cronologías individuales (cuadro 4). Los pilotes del piso de la iglesia de Ichuac ICH-01 e ICH-05 no presentaron corteza y tampoco albura, ambos indicadores de que la fecha de muerte de los árboles que dieron origen a estas piezas de madera fue posterior a la fecha del último anillo presente en las muestras. Los años correspondientes a los últimos anillos encontrados en ICH-01 e ICH-05 fueron 1893 y 1783, respectivamente (cuadro 4). Por su parte, los pilotes de Ichuac ICH-02 e ICH-04 tampoco presentaron corteza, no obstante la presencia de albura indicó que la fecha de muerte del árbol estaría relativamente cercana a la fecha de los

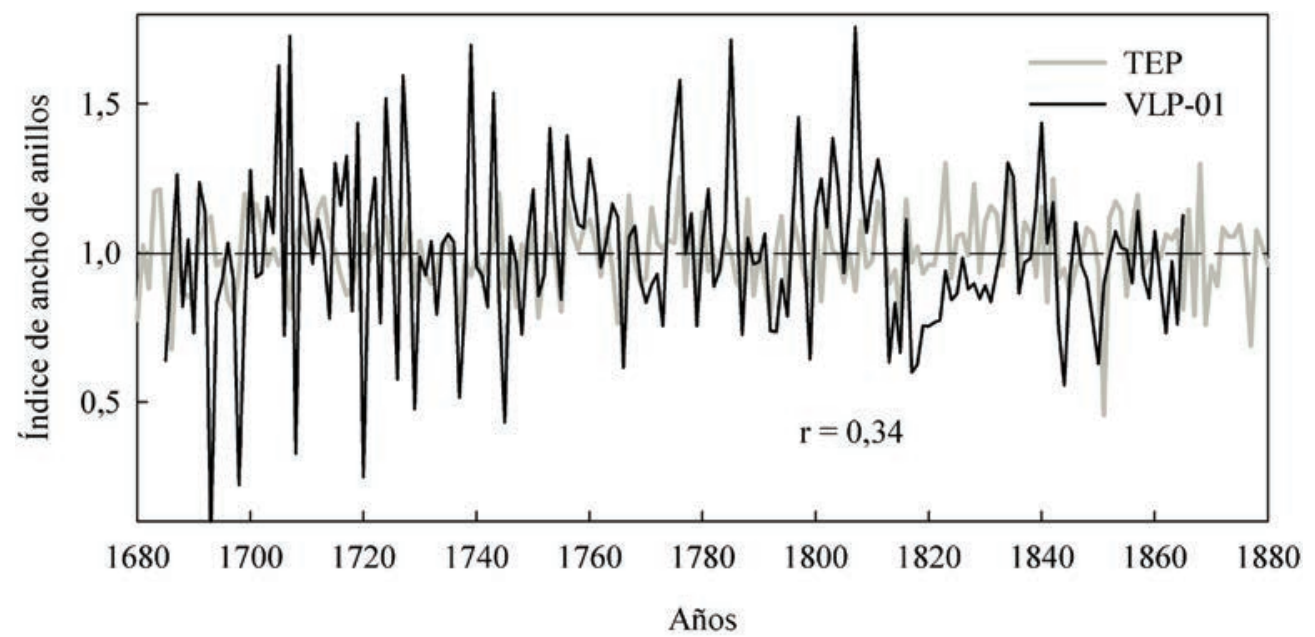

Figura 4. Comparación de series de crecimiento de P. uviferum provenientes del pilar de la torre la Iglesia de Vilupulli VLP-01 (línea negra) con la cronología de Tepuhueico (TEP, línea gris) para el periodo de 1686-1856. Nótese la correspondencia temporal de la variabilidad interanual entre los anillos de crecimiento de la muestra del pilar VLP-01 y la cronología de ancho de anillos de Tepuhueico.

Time-series comparison (Pearson correlation coefficient) between the tower pillar sample VLP-01 from the Vilupulli church (black line) and the P. uviferum chronology at Tepuhueico (TEP) (gray line) for the 1686-1856 period.

Cuadro 4. Características de las dataciones de los pilotes del piso de la Iglesia de Ichuac.

Dating and statistical characteristics from the floor-beam timber section from the Ichuac church.

\begin{tabular}{rcccccc}
\hline Código & $\mathrm{N}^{\circ}$ de años & Año de inicio & Año de término & Anillo externo & Cronología & $\mathrm{r}$ \\
\hline ICH-01 & 97 & 1796 & 1893 & $\mathrm{D}$ & ETP & 0,35 \\
ICH-01 & 97 & 1796 & 1893 & $\mathrm{D}$ & COM & 0,10 \\
ICH-02 & 89 & 1832 & 1920 & $\mathrm{C}$ & ETP & 0,43 \\
ICH-02 & 89 & 1832 & 1920 & $\mathrm{C}$ & TEP & 0,32 \\
ICH-02 & 89 & 1832 & 1920 & $\mathrm{C}$ & COM & 0,24 \\
ICH-04 & 79 & 1850 & 1929 & $\mathrm{C}$ & SLN & 0,32 \\
ICH-04 & 79 & 1850 & 1929 & $\mathrm{C}$ & COM & 0,21 \\
ICH-05 & 135 & 1649 & 1783 & D & ETP & 0,43 \\
ICH-05 & 135 & 1649 & 1783 & D & COM & 0,27 \\
\hline
\end{tabular}


últimos anillos encontrados en cada una, que corresponden a 1920 y 1929, respectivamente (cuadro 4).

Las edades de los pilotes del piso de la iglesia de Ichuac fluctuaron entre 79 y 135 años, y sus correlaciones con distintas cronologías variaron entre $\mathrm{r}=0,32(P<0,05)$ y $\mathrm{r}=0,43(P<0,05)$. La muestra ICH-01, de 97 años, se correlacionó con la cronología de ancho de anillos Piuché (ETP) con un valor de $\mathrm{r}=0.35(P<0,05$; cuadro 4 , figura $5 \mathrm{~A})$.
Por su parte, el pilote de 89 años ICH-02, se correlacionó con dos cronologías distintas, Piuché (ETP) y Tepuhueico (TEP), presentando las siguientes correlaciones $r=$ $0,43(P<0,05)$ y $r=0,32(P<0,05)$, respectivamente, para el mismo periodo (1832-1920, cuadro 4, figura 5B y 5C).

La muestra ICH-04, que abarcó 79 años, se correlacionó con un valor de $\mathrm{r}=0,32(P<0,05)$ con la cronología Santa Lucía (SNL) durante el periodo 1850-1929 (cuadro
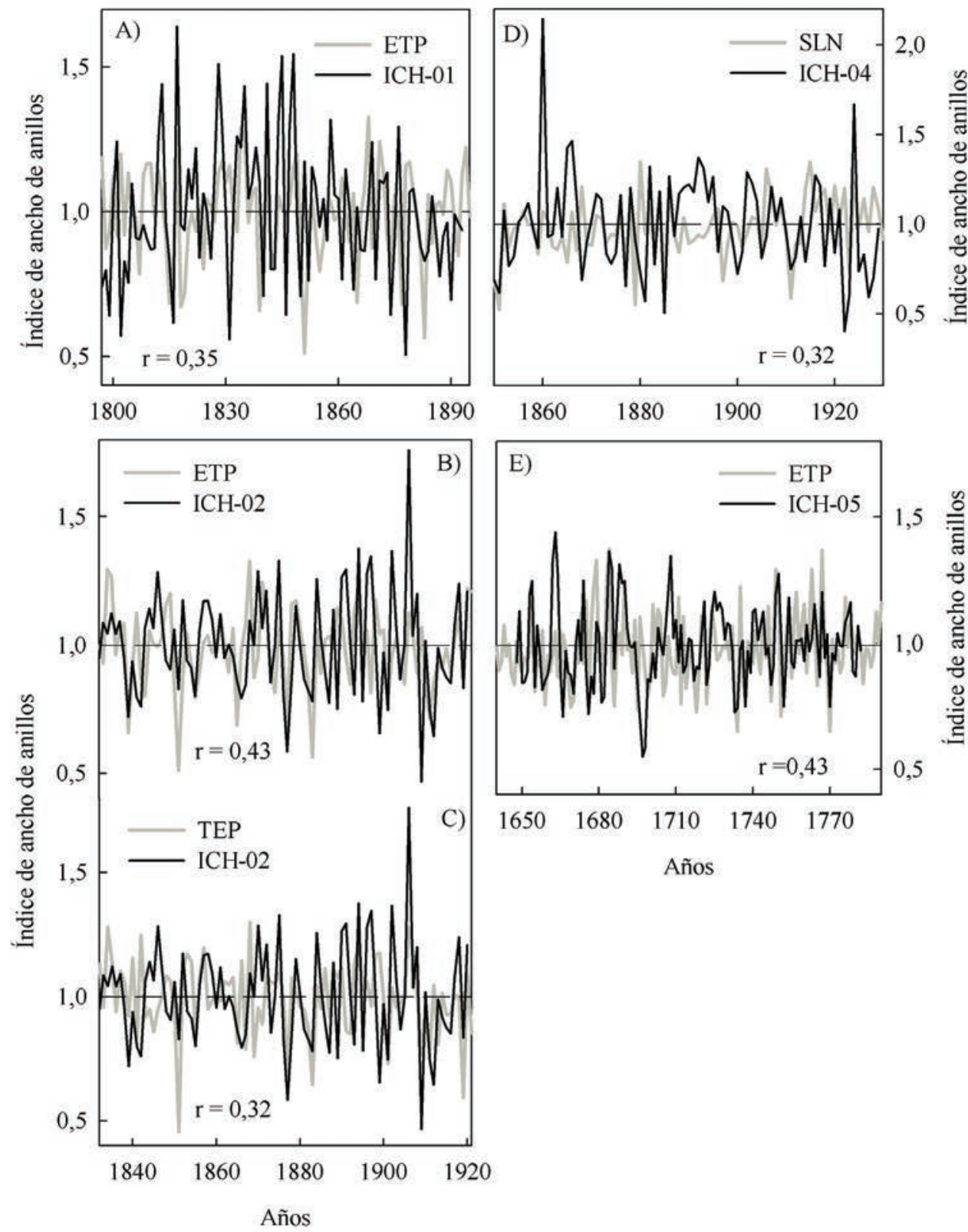

Figura 5. A) Comparación de los patrones de crecimiento de las muestras de las Iglesias (líneas negras) y las cronologías de ancho de anillos de P. uviferum (líneas grises). A) Muestra ICH-01 vs cronología Piuche; B y C) ICH-02 vs. cronologías Piuche y Tepuhueico; D) ICH-04 vs cronología Santa Lucia; E) ICH-05 vs cronología Pichué. Se indican los coeficientes de correlación de Spearman.

Comparison (and Spearman correlation coefficient) between tree-ring width indices from the Ichuac church's floor beam samples (black) and P. uviferum chronology (gray). A) Church sample ICH-01 and the Piuché (ETP) chronology; B) and C) Church sample ICH-02 and the Piuché (ETP) chronology and the Tepuhueico (TEP) chronolog; D) Church sample ICH-04 and the Santa Lucía (SLN) chronology, and E) Church sample ICH-05 and the Piuché (ETP) chronology. 
4, figura 5D). Esta fue la muestra que presentó la menor similitud visual al comparar los crecimientos medidos en los cuatro pilotes con las cronologías de ancho de anillos de $P$. uviferum utilizadas como series de referencia (figura 5D).

La muestra ICH-05 presentó un valor de correlación de $r=0,43(P<0,05)$ con la cronología de ancho de anillos de Piuché (ETP) para el período 1649-1783. Esta muestra contuvo el segmento de mayor cantidad de años (135 años) y mejor valor de correlación, en comparación con las otras muestras de la Iglesia de Ichuac (figura 5E).

\section{DISCUSIÓN}

Potencialidades y limitaciones para el fechado de piezas de madera utilizando Pilgerodendron uviferum. A partir de los resultados obtenidos se puede corroborar que la especie $P$. uviferum posee un alto potencial para datar piezas de madera de las iglesias de Chiloé y otras estructuras patrimoniales en la región. Los patrones de crecimiento encontrados en las piezas de madera de las dos iglesias analizadas fueron similares a aquellos encontrados en las cronologías de ancho de anillos de sitios provenientes de la Isla de Chiloé y de otras zonas cercanas en Chile continental. Análisis a un mayor número de muestras permitirían corroborar las fechas encontradas en estas primeras piezas analizadas dendrocronológicamente en las iglesias de Chiloé.

Las principales limitaciones para el estudio de piezas de madera de $P$. uviferum provenientes de las iglesias analizadas, se vincularon a la escasez de piezas que presentaran corteza o al menos albura, y a la extensión de las series de crecimiento contenidas en las mismas. La presencia de corteza en piezas dendroarqueológicas permite asignar con seguridad la fecha del último anillo de crecimiento en la serie, correspondiente a la fecha en que los árboles murieron o fueron cortados (Kuniholm 2001). Otro aspecto de relevancia para considerar en futuros estudios, es que no todos los bosques de esta especie poseen árboles que presenten albura en forma clara. Por este motivo, es posible que este indicador no sea de total utilidad al momento de determinar la incertidumbre de las fechas encontradas, siendo la presencia de corteza posiblemente el único indicador ciento por ciento fiable en estudios utilizando P. uviferum. Al parecer los individuos jóvenes presentan la albura de forma más clara, mientras que en individuos de mayor edad no siempre es posible diferenciar albura en las muestras. Muestreos en otras iglesias, aun no procesados, han encontrado piezas de madera utilizadas en distintas partes de las estructuras con presencia de corteza. No obstante, las piezas hasta la fecha observadas por los autores parecen siempre provenir de otras especies arbóreas nativas. Sin embargo, aún hay muchas iglesias no estudiadas y que pueden ser visitadas para corroborar la presencia de corteza en piezas de madera de P. uviferum y otras especies en futuros estudios. Por otro lado, la segunda limitación de importancia para la datación de algunas de las piezas utilizadas en este estudio, fue la corta extensión de las series de crecimiento encontradas en algunas secciones de las iglesias, como fue el caso de los pilotes de la Iglesia de Ichuac. Lo anterior aumenta la incertidumbre de las dataciones dado que la correspondencia temporal se considerada más robusta si ocurre a lo largo de una amplia extensión de tiempo. Cuando la serie de crecimiento que se quiere fechar es una serie corta, por ejemplo menor a 100 años, hay más probabilidad que su patrón de crecimiento coincida con los patrones de crecimiento en diferentes secciones de la serie de referencia, mientras que si la serie a fechar es larga, esa probabilidad disminuye y aumenta la seguridad de la datación (Barclay y Rayburn 2014). Por ejemplo, una datación dendroarqueológica de una casa histórica en Nueva York, resultó en que las muestras de tablas de madera con menos de 40 anillos de crecimiento no pudieron ser datadas, sugiriendo que para una exitosa datación cruzada la muestra debe ser superior a 100 años (Barclay y Rayburn 2014). Como en el caso de estudio de Barclay y Rayburn (2014), este problema puede ser también resuelto en futuras investigaciones en las iglesias de Chiloé, utilizando una mayor cantidad de muestras y extendiendo el muestreo a una mayor cantidad de secciones o partes de las estructuras dentro de las iglesias.

Fechado de las piezas de madera de P. uviferum de las iglesias de Ichuac y Vilupulli. Uno de los principios fundamentales de una datación dendrocronológica consiste en que las muestras que se buscan datar pueden presentar un patrón de crecimiento similar a otros árboles de la misma especie en una zona geográfica (Holmes 1983). Esto sucede debido a que tanto los árboles que dieron origen a la estructura, como los que fueron incluidos en las cronologías de ancho de anillos, comparten un patrón de crecimiento que es normalmente atribuido a las variaciones del clima de la zona, el cual condiciona el crecimiento de los árboles en grandes escalas geográficas; es decir, más allá de su condición local de sitio (Kuniholm 2001). Considerando el desconocimiento acerca de la procedencia y el año de instalación de las piezas de madera en las iglesias, se utilizaron todas las cronologías de ancho de anillos disponibles de la especie en la zona para evaluar su relación con cada pieza de madera, incluyendo también una cronología compuesta por series de las tres mejores cronologías individuales en cuanto a correlaciones con las muestras de las iglesias (cuadro 2). Debido a que aún existen pocas cronologías de ancho de anillos de esta especie en Chiloé, es posible que al mejorar la red de cronologías de referencia para la datación de estructuras históricas en la isla y sus alrededores, se pueda también mejorar las correlaciones y la seguridad de la datación de cada pieza. En ninguno de los casos las correlaciones de las muestras analizadas con las series de referencia $(r=0,37 ; P<0,05$; cuadros 3 y 4$)$ alcanzaron los valores de intercorrelación de las cronologías ( $\mathrm{r}=0,56$ en promedio; $P<0,05$; cuadro 2 ), sugiriendo que otras cronologías, posiblemente de sitios más próximos o similares a los que dieron origen a los árboles usados en la 
construcción de las iglesias de Chiloé, podrían mejorar las correlaciones entre piezas y series de referencia, y junto con esto mejorar también la certeza de las dataciones. La ubicación de los sitios y la complacencia o restricciones para el crecimiento de los mismos, son consideradas posibles causas de las bajas correlaciones entre los patrones de crecimiento de las piezas de madera de la iglesia de Ichuac con las cronologías o series de referencia utilizadas. Generalmente, las cronologías de ancho de anillos provienen de sitios restrictivos para el crecimiento, en donde se expresan de mejor forma las diferencias interanuales en las series de crecimiento de los árboles. Esta condición es especialmente deseada para estudios en dendroclimatología, pero podría no ser el caso de los sitios que dieron origen a los árboles utilizados en la construcción de las iglesias de Chiloé.

Iglesia de Vilupulli. Considerando que la iglesia de Vilupulli se comenzó a construir a principios del siglo XX (Guarda 1984, Montecinos et al. 1995), es posible que el pilar VLP-05 proveniente de la torre, que presentó el año 1918 como el más reciente en la muestra, sea parte del proceso tardío de la construcción inicial o de una restauración no descrita en documentación histórica. A pesar de que VLP-05 no presentaba corteza, indicador que permite tener seguridad del año en que fue cortado el árbol, esta muestra presentaba albura en forma clara. Sin embargo, considerando que el número de anillos presentes en la albura puede estar entre 20 y 50 en algunas cronologías, con un promedio de 30 años, es también posible que la fecha de corte se encuentre varios años posterior a 1918. En el caso de la muestra VLP-01, que presentó como anillo más reciente el año 1866, se evidenció un fuerte labrado y ausencia de albura, lo cual pudo hacerle perder varios segmentos de anillos de crecimiento que podrían ser cercanos a la fecha obtenida para VLP-05 (año 1918). No obstante, no se puede descartar que VLP-01 sea una pieza más antigua, quizás utilizada en las etapas iniciales de construcción de la iglesia. El análisis de los otros pilares y otras piezas de la torre podrían ayudar a precisar las fechas de construcción y restauración de la torre de esta iglesia. La datación de piezas de otras especies que se observan con mayor frecuencia siendo usadas en forma rústica y con presencia de corteza, podría ser de utilidad para disminuir la incertidumbre de la datación de las estructuras dentro de las iglesias de la Isla de Chiloé. En algunos casos, el proceso de construcción de una iglesia en Chiloé pudo durar diez o más años (Montecinos et al. 1995). Este antecedente es de primera importancia para evaluar la antigüedad de las piezas de madera y el rango en que la datación dendroarquelógica puede ser asignada a un determinado momento de la historia. Este rango de tiempo, posiblemente mayor o menor a diez años en cada iglesia, debería considerarse en futuros estudios dendroarquelógicos, dado que limita la posibilidad de determinar si la pieza fue cortada mientras se construía cada iglesia, o si era una pieza cortada con poca o mucha anterioridad.
Iglesia de Ichuac. La iglesia de Ichuac fue construida a finales del siglo XIX (Guarda 1984), lo cual indicaría que las dataciones de los últimos anillos de los pilotes (ICH02: 1920; ICH-04: 1929), con presencia de albura, formarían parte de una restauración no descrita anteriormente. Es importante mencionar que se desconocen restauraciones anteriores a la fecha del terremoto de 1960, después del cual se habría restaurado esta y varias otras iglesias de la isla, resaltando la necesidad de reconstruir la historia de estas y otras estructuras presentes en la zona.

Los pilotes ICH-01 e ICH-05 no presentaron corteza ni albura, por ende, no fue posible asignar el año de corte del árbol que dio origen a estas piezas de madera. No obstante, dado que ambos pilotes presentaron un fuerte labrado, es posible que las piezas hayan perdido varios anillos exteriores. Una mayor cantidad de muestras de los pilotes, idealmente de mayor longitud temporal, provenientes de la estructura del piso de la iglesia de Ichuac permitirán corroborar las dataciones encontradas en este estudio. Existe un alto potencial de encontrar piezas rústicas de madera en las iglesias de Chiloé, con albura y corteza, es decir, sin labrado. Esto es más probable en las estructuras del piso y las fundaciones, como en pilares y algunas vigas utilizadas en el techo y la torre. De identificar este tipo de muestras, el fechado podría llegar a ser incluso exacto respecto del año en el cual fueron cortados los árboles utilizados en las estructuras de las iglesias de Chiloé.

La evidencia de que las muestras de las dos iglesias, Vilupulli e Ichuac, analizadas en este estudio, correlacionan con las cronologías de ancho de anillo Piuché (ETP), Santa Lucía (SLN) y Tepuhueico (TEP), se puede atribuir a que comparten una señal de crecimiento potencialmente explicada por la variabilidad climática común. Según Aravena y Luckman (2009) la Isla de Chiloé posee un clima similar en comparación con otras zonas del sur de Sudamérica. Corroborando esto, las tres cronologías se correlacionaron con la precipitación $(\mathrm{r}=0,48 ; P<0,05 ; \mathrm{n}=47)$ y la temperatura media $(\mathrm{r}=-0,49 ; P<0,05 ; \mathrm{n}=47)$ de los meses de primavera de la isla de Chiloé. Resultados similares, incluyendo también la variabilidad climática de los meses de verano y otoño, han sido encontrados en otros estudios analizando las relaciones clima-crecimiento en $P$. uviferum (Lara et al. 2008).

Potencial de la dendroarqueología en el bosque templado Iluvioso de Chile y Argentina. Debido a que la madera de $P$. uviferum presenta un crecimiento recto y su madera posee una alta durabilidad (Solis et al. 2004), ha sido una de las especies más utilizadas en el sur de Chile como revestimiento, postes, pilares y vigas (Bannister et al. 2008). En todos estos casos, las piezas de madera pueden ser utilizadas en forma rústica, incluso sin ningún labrado, favoreciendo la identificación de los años en los cuales los árboles han sido cortados, lo cual en muchos casos puede estar muy próximo a la fecha de construcción de las estructuras. En este sentido, la integración de esta información con el 
conocimiento local e histórico de construcción en madera, puede ayudar a lograr una mejor estimación de la fecha de construcción o restauración de las estructuras. La excepcionalidad de esta especie en cuanto a las características de su madera y su durabilidad (Solis et al. 2004), su sensibilidad climática (Lara et al. 2008) y su potencial para datar estructuras y piezas de madera, ratifican la necesidad de expandir el estudio de los anillos de crecimiento de los bosques de P. uviferum como información clave para el patrimonio, la historia cultural y ambiental de Chiloé y sus alrededores.

Existe una cantidad creciente de especies que han mostrado ser de utilidad para estudios dendrocronológicos en bosques templados de Chile y Argentina. A pesar de esto, varias de las especies que fueron encontradas durante los procesos de restauración de las iglesias de Chiloé, no han sido nunca puestas a prueba de un modo sistemático dentro de esta disciplina. Algunas de ellas, como Weinmania trichosperma Cav. (tineo), Drymis winteri J.R. et G. Forster (canelo), Nothofagus nitida (Phil) Krasser (coihue de Chiloé), especies de mirtáceas y Laurelipsis phillipiana Looser (tepa), entre otras, se han hallado en iglesias restauradas recientemente. La presencia de una gran cantidad de piezas de L. phillipiana en la iglesia de Santa María de Rilán, especie que no se utiliza actualmente en construcción por sus características físicas, organolépticas y su durabilidad, abre fuertes interrogantes acerca de la conservación del conocimiento de construcción y de los atributos de las especies del bosque y sus usos en Chiloé. Una mayor cantidad de estudios dendrocronológicos en nuevas especies del bosque templado lluvioso favorecerá el estudio de estas y otras estructuras históricas construidas hace más de cien años.

Un número mayor de estudios sobre registros históricos de las estructuras (restauraciones, remodelaciones, incendios, etc.), junto con el conocimiento histórico de las técnicas de construcción (tiempo de secado, formas de uso de cada especie), permitirán integrar y contextualizar la información histórica que puede proveer el estudio de los anillos de crecimiento como método de datación de las piezas de madera de las iglesias. Otras especies que han sido halladas en construcciones históricas en Chiloé podrían también ser estudiadas en cuanto a su potencial dendrocronológico, considerando que muchas de estas especies poseen una alta durabilidad y aparente buena visibilidad de anillos de crecimiento.

\section{CONCLUSIONES}

Pilgerodendron uviferum tiene un alto potencial para estudios dendroarqueológicos en estructuras patrimoniales del sur de Chile. Los resultados de este estudio ofrecen dataciones de las piezas de madera dentro de los rangos históricos esperados y con buenos parámetros estadísticos utilizando tres cronologías de ancho de anillos de la especie como series de referencia. No obstante lo anterior, un mayor número de piezas de madera permitiría corroborar las fechas propuestas en este estudio.
Los resultados positivos de este estudio exploratorio, datando un número reducido de muestras de $P$. uviferum, resaltan la necesidad de continuar con la datación de más estructuras y piezas de madera de las iglesias de Chiloé y otras construcciones consideradas patrimoniales, con el fin de reconstruir la historia de estas edificaciones, en lo que podría ser uno de los lugares más húmedos del mundo donde se puede datar madera después de siglos de haber sido extraída del bosque.

\section{AGRADECIMIENTOS}

Agradecemos al proyecto "Potencial de los anillos de crecimiento de árboles para la datación de estructuras Históricas (Proyecto DID S-2013) de la Dirección e Investigación de la Universidad Austral de Chile. También agradecemos el aporte de National Geographic Society (fondo \#7988-06), National Science Foundation (fondos \# 0602166 and 0956552), Centro del Clima y la Resiliencia CR2 (FONDAP 15110009), proyecto Fondecyt 11140677 y a los Proyectos Internos Pontificia Universidad Católica de Valparaíso (039.353/2016 and 039.329/16).

Por su apoyo en la colecta de muestras, información histórica y geográfica agradecemos a Gabriel Guarda, Álvaro González, Elizabeth Uribe, Natalia Riquelme, Katrin Fitzek, Hermes Vera y Patricio Basáez. Agradecemos ampliamente la participación de la Fundación Amigos de las Iglesias de Chiloé por facilitar diversas instancias de muestreo y obtención de información histórica para el desarrollo de esta investigación. Finalmente agradecemos a tres revisores anónimos que con sus comentarios ayudaron a mejorar la calidad de este trabajo.

\section{REFERENCIAS}

Aravena JC, BH Luckman. 2009. Spatio-temporal rainfall patterns in southern South America. International Journal of Climatology 29(14): 2106-2120.

Baillie MGL. 1995. A Slice Through Time: Dendrochronology and Precision Dating. Batsford, London, UK. Routledge. $176 \mathrm{p}$.

Bannister J, C Le Quesne, A Lara. 2008. Estructura y dinámica de bosques de Pilgerodendron uviferum afectados por incendios catastróficos en la Cordillera de la Costa de la Isla Grande de Chiloé. Bosque 29(1): 33-43.

Bannister J. 2012. Dynamics and restoration of Pilgerodendron uviferum forests on Chiloé Island, North Patagonia, Chile. Thesis PhD. Freiburg im Breisgau, Germany. Faculty of Forest and Environmental Sciences, Albert-Ludwigs Universität. $93 \mathrm{p}$.

Barclay DJ, JA Rayburn. 2014. Tree-ring dating of historic buildings in Willsboro, northeastern New York, and development of regional chronologies for dendroarchaeology. TreeRing Research 70(2): 79-90.

Berg L. 2007. Restauración en Chiloé (Chile): la Iglesia de Castro. Apuntes 20(1): 126-141.

Briffa K. 1995. Interpreting high-resolution proxy climate data: the example of dendroclimatology. In Von Storch H, A Na- 
varra eds. Analysis of climate variability, applications of statistical techniques. Berlin, Germany. Springer. p. 77-94.

Cook E, LA Kairiukstis. 1990. Methods of Dendrochronology: Applications in the Environmental Sciences. New York, USA. Springer Science \& Business Media. 394 p.

Di Castri F, ER Hajek. 1976. Bioclimatología de Chile. Santiago, Chile. Vicerrectoría Académica, Universidad Católica de Chile. 107 p.

Donoso C, A Lara, B Escobar, A Premoli, C Souto. 2013. Fitzroya cupressoides (Molina) I. M. Johnst. Alerce. In Donoso Zegers $\mathrm{C}$ ed. Las especies arbóreas de los bosques templados de Chile y Argentina. Autoecología. Valdivia, Chile. Marisa Cuneo Ediciones. p. 68-81.

Fundación Amigos de las Iglesias de Chiloé. 2015. Patrimonio de la Humanidad. Consultado el 15 mar. 2015. Disponible en http://www.iglesiasdechiloe.cl/?page_id=39.

Guarda G. 1984. Las Iglesias de Chiloé. Santiago, Chile. Ediciones Universidad Católica de Chile. 236 p.

Holmes RL. 1983. Computer-assisted quality control in tree-ring dating and measurements. Tree Ring Bulletin 43: 69-75.

Holz A, T Veblen. 2011. The amplifying effects of humans on fire regimes in temperate rainforests in western Patagonia. $\mathrm{Pa}$ laeogeography, Palaeoclimatology, Palaeoecology 311(12): 82-92.

ITRDB (International Tree-Ring Data Bank, US). 2014. Tree Ring. Consultado 30 Ago. 2014. Disponible en https:// www.ncdc.noaa.gov/data-access/paleoclimatology-data/ datasets/tree-ring.

KNMI (Koninklijk Nederlands Meteorologisch Instituut, NL). 2015. Monthly Observations. Consultado el 20 mar. 2015. Disponible en http://climexp.knmi.nl/selectfield_obs2. cgi?id=someone@somewhere.

Kuniholm PI. 2001. Dendrochronology and Other Applications of Tree-ring Studies in Archaeology. In Brothwell DR, AM Pollard eds. The Handbook of Archaeological Sciences, London, UK. John Wiley. p. 36-46.

Lara A, R Villalba, R Urrutia. 2008. A 400-year tree-ring record of the Puelo River summer-fall streamflow in the Valdivian Rainforest eco-region, Chile. Climatic Change 86(3): 331-356.

Lara A, J Bannister, C Donoso, A Rovere, D Soto, B Escobar, A Premoli. 2013. Pilgerodendron uviferum (D. Don) Florin.
Ciprés de las Guaitecas. In Donoso Zegers C ed. Las especies arbóreas de los bosques templados de Chile y Argentina. Autoecología. Valdivia, Chile. Marisa Cuneo Ediciones. p. 82-92.

Montecinos H. 1995. Las iglesias misionales de Chiloé. Santiago, Chile. Facultad de Arquitectura y Urbanismo, Universidad de Chile. 117 p.

Morales MS, AE Nielsen, R Villalba. 2013. First dendroarchaeological dates of prehistoric contexts in South America: chullpas in the Central Andes. Journal of Archaeological Science 40(5): 2393-2401.

Nash SE. 1999. Time, Trees, and Prehistory: Tree-Ring Dating and the Development of North American Archaeology, 1914-1950. Salt Lake City, USA. University of Utah Pres. $294 \mathrm{p}$.

Rivera MA, J Moya, D Shea. 2010. Dendrocronología en la Pampa del Tamarugal Desierto de Atacama, Norte de Chile. Diálogo Andino - Revista de Historia, Geografía y Cultura Andina 36: 33-50.

Sahady A, F Gallardo, J Bravo, M Ibañez. 2007. Las unidades espaciales asociadas a la religiosidad chilota: Evolución de las entidades pobladas. Santiago, Chile. Instituto de Restauración Arquitectónica, Facultad de Arquitectura y Urbanismo, Universidad de Chile. 12 p.

Slayton JD, MR Stevens, HD Grissino-Mayer, CH Faulkner. 2009. The historical dendroarcheology of two log structures at the Marble Springs Historic Site, Knox County, Tennessee, U.S.A. Tree-ring Research 65(1): 23-36.

Solís C, J Becerra, C Flores, J Robledo, M Silva. 2004. Antibacterial and antifugal terpenes from Pilgerodendron uviferum (D. Don) Florin. Journal of the Chilean Chemical Society 49(2): 157-161.

UNESCO (United Nations Educational, Scientific and Cultural Organization, FR). 2003. Convention Concerning the Protection of the World Cultural and Natural Heritage. Properties inscribed on the World Heritage List. Paris, France. UNESCO. 179 p.

Veblen TT, JJ Armesto, BR Burns, T Kitzberger, A Lara, B León, K Young. 2005. The Coniferous Forest of South America. In Anderson F ed. Ecosystems of the World: Coniferous Forest. Amsterdam, The Netherlands. Elsevier. p .702-725. 
